\title{
Josephson nonequilibrium phenomena under current injection in superconducting three-terminal stacked devices
}

\author{
G. P. Pepe, G. Peluso, R. Scaldaferri, and A. Barone \\ INFM Coherentia-Università di Napoli "Federico II," Dip. Scienze Fisiche, Fac. Ingegneria, Ple Tecchio, 80-I-80125 Naples, Italy \\ L. Parlato \\ Seconda Università degli Studi di Napoli, Facoltà di Ingegneria, Via Roma 29, Aversa (CE), Italy
}

R. Latempa

INFM-Università di Salerno, I-84081 Baronissi, Italy

A. A. Golubov

University of Twente, Department of Applied Physics, P.O. Box 217, 7500 AE Enschede, The Netherlands

(Received 3 May 2002; revised manuscript received 12 July 2002; published 12 November 2002)

\begin{abstract}
Three-terminal superconducting tunnel devices offer a powerful tool to investigate nonequilibrium phenomena in superconductors. We study the role of the proximity effect in a $\mathrm{Nb} / \mathrm{Al}$ bilayer forming the intermediate electrode of a stacked structure configuration. The spatial profile of the pair potential along the common electrode and the effective density of states near the detector tunnel barrier are determined by combining the deconvolution of the current-voltage $(I-V)$ injector characteristics and the numerical solution of the microscopic proximity model equations. The Josephson current variation following a steady-state injection was measured down to $1.5 \mathrm{~K}$. Results are discussed in the framework of a simple nonequilibrium model, which leads also to an estimate of the effective relaxation time limiting quasiparticle scattering processes in the $\mathrm{Nb} / \mathrm{Al}$ bilayer.
\end{abstract}

DOI: 10.1103/PhysRevB.66.174509

PACS number(s): 74.50.+r, 85.25.-j, 72.20.Jv

\section{INTRODUCTION}

In recent years double-barrier stacked structures, formed by two Josephson junctions vertically grown with a common middle electrode, have received great attention. In particular, the large variety of new physical phenomena exhibited by such configurations triggered the attention toward potential applications in the realization of new transistor-like devices ${ }^{1}$ as well as in the improvement of existing fluxon oscillators. ${ }^{2}$ Moreover, they offer a powerful tool for the understanding of nonequilibrium phenomena in superconductors. Relaxation processes involving quasiparticles, phonons, and Cooper pairs have been successfully investigated in different materials and/or device configurations. ${ }^{3}$ Many advantages from these studies both in basic physics and high performance electronics were achieved.

In a three-terminal configuration the electrodes are connected in such a way that the two junctions are independently biased. Quasiparticles are injected into the common electrode by biasing one of the two junctions of the stack (injector) at a voltage larger than the sumgap value. The excess of injected electronic excitations reflects in a variation of the current-voltage $I-V$ characteristics of the other junction (detector) thus yielding information on both quasiparticles and phonons relaxation mechanisms. The Josephson current is also affected by this nonequilibrium state, since any change in the electron-phonon interaction modifies both the energy gap and the electronic distribution function. An extra current in one of the two electrodes produces primary electronic excitations at an energy $E \approx \Delta$, i.e., the limiting energy in the perturbed material. A number of these injected quasi- particles recombines to form Cooper pairs again through the emission of $2 \Delta$ phonons. If the energy of injected quasiparticles is higher than the gap edge of the intermediate electrode, high energy phonons produced by recombination processes can break Cooper pairs, creating two more quasiparticles. The dependence of the Josephson current on the released energy has been proposed for new superconducting high sensitivity particle detectors. ${ }^{4}$ In order to investigate the quasiparticle relaxation we fabricated three-terminal stack superconducting double tunnel junctions in which the common electrode was formed by a Nb/Al bilayer. The bottom junction was used to inject electronic excitations whose effects were measured through the detector Josephson current variation. The inductive (or magnetic) coupling between the two junctions of the stack, as a consequence of the screening currents in the common electrode when its thickness is about equal or smaller than the London penetration depth $\lambda_{L}$, is the dominant effect in these type of measurements, especially in the case of large Josephson junctions, where the characteristic length is larger than $\lambda_{L}$. However, the expected saturation of $\lambda_{L}$ at low temperatures $\left(k_{B} T\right.$ $\ll \Delta$ ) suggests the nonequilibrium contributions can be successfully evidenced in Josephson critical current $I_{c}$ vs injection current $I_{\text {inj }}$ patterns.

In this paper we report measurements of the maximum supercurrent in three terminal devices consisting of two tunnel stacked Josephson junctions. The intermediate electrodes of the investigated samples consisted of a bilayer $\mathrm{Nb}-\mathrm{Al}$ heterostructure close to the detector barrier. This choice was suggested by the possibility to enhance the confinement of quasiparticles in the $\mathrm{Al}$ volume just close to the detector 
tunnel barrier by means of the trapping effect. ${ }^{5}$ The presence of inhomogeneities in the pair potential profile along the bilayer due to the proximity coupling between $\mathrm{Nb}$ and $\mathrm{Al}$ was also taken into account. $I_{c}$ vs $I_{\text {inj }}$ experimental patterns were measured down to $T=1.5 \mathrm{~K}$. The nonequilibrium contribution to the Josephson current change was isolated by means of a subtraction of the residual magnetic contribution at low temperatures. An estimation of the most limiting quasiparticle lifetime was also achieved, and it is in agreement with nonequilibrium theories in proximised bilayers.

In Sec. II we present experimental results concerning both the fabrication and the characterization of stack tunnel devices. The modifications of the Josephson current $I_{c}$ in the presence of a steady-state injection current $I_{\text {inj }}$ across the injector at different temperatures are presented. The spatial profile of the pair potential along the common electrode and the effective density of states (DOS) near the detector tunnel barrier are determined by combining the deconvolution of current-voltage $I-V$ injector characteristics and the numerical solution of the microscopic proximity model equations. The analysis of the proximity effect in the presence of a steadystate current injection is important in order to understand the nature of the involved nonequilibrium processes. In Sec. III a discussion about possible mechanisms which can influence the $I_{c}$ vs $I_{\text {inj }}$ measurements is proposed. A simple nonequilibrium model for the Josephson current in the presence of a steady-state current injection is also proposed. According to this model an estimation of the effective quasiparticle lifetime is produced. The analysis suggests the quasiparticle trapping in the $\mathrm{Nb}$ side of the intermediate electrode is the most effective process limiting excitation lifetimes. The agreement with theoretical predictions is an indirect demonstration of the validity of the proposed nonequilibrium analysis.

\section{EXPERIMENTAL}

\section{Fabrication}

The multilayer $\mathrm{Nb}(150 \mathrm{~nm}) / \mathrm{Al}_{x} \mathrm{O}_{y} / \mathrm{Nb}(40 \mathrm{~nm})$ $\mathrm{Al}(20 \mathrm{~nm}) / \mathrm{Al}_{x} \mathrm{O}_{y} / \mathrm{Nb}(50 \mathrm{~nm})$ was deposited over a 2 -in. $\mathrm{Si}$ crystalline wafer in a ultrahigh vacuum system at pressure of $10^{-8}$ Torr, without vacuum breaking. ${ }^{6}$ This process allows a high degree of control for interfaces between different layers. $\mathrm{Nb}$ and $\mathrm{Al}$ films were deposited by dc-magnetron sputtering while tunneling barriers were formed by Al thermal oxidation. The multilayer was patterned by using the lift-off technique to define the geometry of the bottom electrode. By dry and chemical etching processes, the top $\mathrm{Nb}$ film, the $\mathrm{Al}_{x} \mathrm{O}_{y}$ oxide and the $\mathrm{Al}$ film were removed in sequence to define the geometry of the top junction. The area of the bottom junction was obtained by anodization oxide growth down to the $\mathrm{Nb}$ of the base electrode. Afterward, an insulation layer of $\mathrm{SiO}$ was thermally deposited on the periphery of the top tunnel barrier, before the deposition of the $\mathrm{Nb}(350 \mathrm{~nm})$ wiring for both the intermediate and top electrode. Finally, the geometry of the bottom lead was defined both by RIE and etching of the bottom tunnel junction. Bottom and top junctions had areas of $108 \times 125$ and $100 \times 100 \mu \mathrm{m}^{2}$, respectively. A cross section of the realized device is shown in Fig. 1.

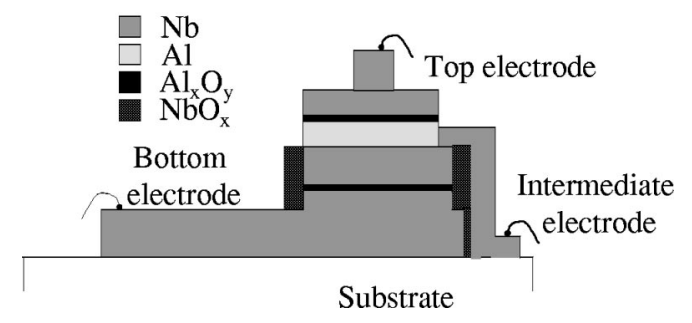

FIG. 1. A schematic of the cross section of a realized device.

\section{Measurements}

The samples were characterized in terms of the currentvoltage $I$ - $V$ curves down to $1.5 \mathrm{~K}$ in a shielded cryostat. Bottom junctions showed high quality factor $V_{m}$ $=0.7 \Delta I_{g} R_{2 \mathrm{mV}}\left(-\Delta I_{g}\right.$ is the current jump at the sum gap voltage and $R_{2 \mathrm{mV}}$ the static resistance at $V=2 \mathrm{mV}$ ) up to 65 $\mathrm{mV}$. The $R_{N N} A$ products were $3.1 \times 10^{-5}$ and 2.6 $\times 10^{-5} \Omega \mathrm{cm}^{2}$ for the bottom and the top junctions, respectively. The electronic properties of the top junctions are strongly influenced by the proximity effect in the intermediate electrode. In fact, the voltage value corresponding to the sumgap was found to be $V_{g}=2.3 \mathrm{mV}$, while for bottom junctions $V_{g}=2.7 \mathrm{mV}$. The current density was $80 \mathrm{~A} / \mathrm{cm}^{2}$. The Josephson critical currents of both junctions were measured as a function of an externally applied magnetic field parallel to the barrier junction. ${ }^{6}$ The diffraction patterns are regular, and they exhibit a good modulation of the $I_{c}$ current up to relatively high magnetic fields. Figure 2 shows the dependence of the Josephson current $I_{c}$ of the top junction on the steady-state current injected from the bottom junction at different temperatures down to $1.5 \mathrm{~K}$. The $I_{c}$ vs $I_{\text {inj }}$ dependence is due to the combined action of the local magnetic field produced by injected currents and nonequilibrium processes. Nevertheless, the saturation observed at temperatures close to $2 \mathrm{~K}$ reflects the achieved insensibility of the magnetic penetration depth at $T \ll T_{c} .{ }^{7}$ The curve recorded at $T=2 \mathrm{~K}$ can be considered as a background, and hence subtracted to lower temperature patterns. Any further change observed in this regime can be a good candidate for the nonequilibrium signature we are searching for. On the other hand, the above-

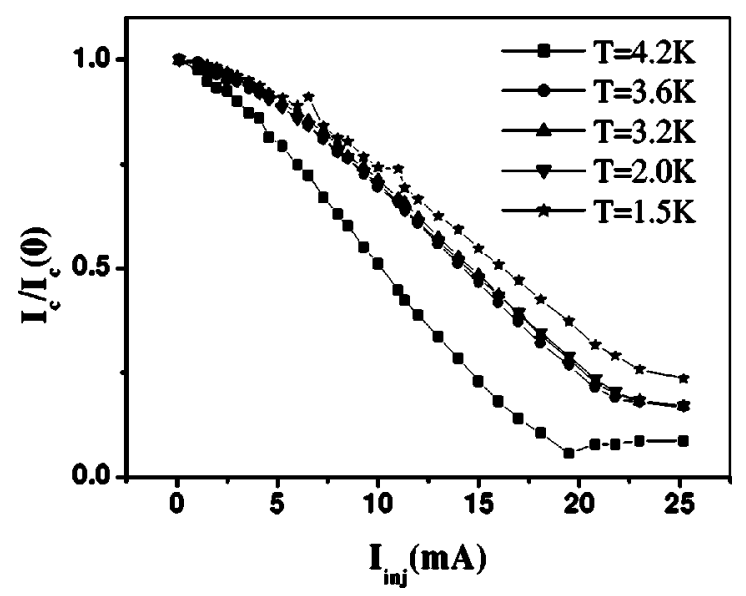

FIG. 2. Modifications in the Josephson current $I_{c}$ under steadystate current injection at different temperatures down to $1.5 \mathrm{~K}$. 
mentioned magnetic background subtraction shouldn't affect seriously nonequilibrium processes, since they are mainly effective at very low temperatures as discussed in the follows.

\section{Injection effects in the proximised $\mathrm{Nb} / \mathrm{Al}$ electrode}

The knowledge of both the effective electronic density of states and the spatial variation of the pair potential along the intermediate electrode are strongly dependent on the proximity coupling between the $\mathrm{Nb}$ and $\mathrm{Al}$ layers forming the intermediate heterostructure. We described the physical properties of the $\mathrm{Nb} / \mathrm{Al}\left(S / S^{\prime}\right)$ bilayer by using a microscopic model proposed by Golubov et al., ${ }^{8}$ which mainly assumes the dirty limit for involved superconductors and an arbitrary finite transparency of the bilayer interface. According to the formalism of Ref. 8, the properties of the $S / S^{\prime}$ bilayer can be described in terms of two physical parameters, namely, $\gamma_{m}$ and $\gamma_{B}$, defined as $\gamma_{m}=\left(\rho_{S} \xi_{S} / \rho_{S^{\prime}} \xi_{S^{\prime}}^{*}\right) d / \xi^{*}$ and $\gamma_{B}$ $=\left(R_{B} / \rho_{S^{\prime}} \xi_{S^{\prime}}^{*}\right)$, where $\rho_{S\left(S^{\prime}\right)}$ is the normal state resistivity of $S\left(S^{\prime}\right)$ layer, $\xi^{*}$ is related to the bulk coherence length $\xi_{S^{\prime}}$, by the relation $\xi_{S^{\prime}}^{*}=\xi_{S^{\prime}} \sqrt{T_{c, S^{\prime}} / T_{c, S}}, \quad \xi_{S}$ is the coherence length of the superconductor $S, d$ the thickness of the $S^{\prime}$ layer, and $R_{B}$ the $S / S^{\prime}$ interface resistivity, respectively. The parameters $\gamma_{m}$ and $\gamma_{B}$ have simple physical meanings: $\gamma_{m}$ is a measure of the strength of the proximity effect between $S$ and $S^{\prime}$ layers, whereas $\gamma_{B}$ describes the effect of the boundary transparency between them.

The variation of the density of states in the superconducting film due to the proximity effect influences also the Josephson current. Indeed, under the assumption of a uniform current distribution across the junction area (small junction limit), the Josephson current can be written as

$$
\frac{e I_{c} R_{N}}{2 \pi T_{c}}=\frac{T}{T_{c}} \sum_{\omega>0} \omega^{-2} \Phi_{1}(0) G_{1}(0) \Phi_{2}(0) G_{2}(0),
$$

where $G_{1(2)}(0)$ is the retarded Green's function for the electrode 1(2) evaluated near the tunnel barrier, $\Phi_{1(2)}(0)$ the pair potential of the electrode 1(2) evaluated at the $S / S^{\prime}$ interface, and $\omega$ is the Matsubara frequency. Taking into account the proximity effect, both the functions $G(0)$ and $\Phi(0)$ are influenced by the parameters $\gamma_{m}$ and $\gamma_{B}$ as explained in Ref. 8 .

A deconvolution technique based on an algorithm proposed by Blamire ${ }^{9}$ allowed us to extract the unknown tunneling density of states (TDOS) from the current-voltage $I-V$ data of the bottom junctions, where sharply peaked gap edges were observed at energies $e V=\Delta+\Delta_{S N}$, with $\Delta$ and $\Delta_{S N}<\Delta$ the energy gaps of the $\mathrm{Nb}$ base and the $\mathrm{Nb} / \mathrm{Al}$ bilayer at the $\mathrm{Nb}$ side electrodes, respectively. Afterward, the extracted TDOS of the intermediate electrode was fitted with that predicted by the microscopic proximity model in the $\mathrm{Nb}$ side of the bilayer by considering $\gamma_{m}$ and $\gamma_{B}$ as free parameters. The best fit values for $\gamma_{m}$ and $\gamma_{B}$ were $\gamma_{m}=0.3$ and $\gamma_{B}=5.2$, respectively. Figure 3 shows the DOS and TDOS of the intermediate electrode on the $S$ side. The BCS density of states has been plotted for clarity. The normalized DOS in the $\mathrm{Al}$ side and the pair potential profile along the intermediate electrode were determined by solving the microscopic

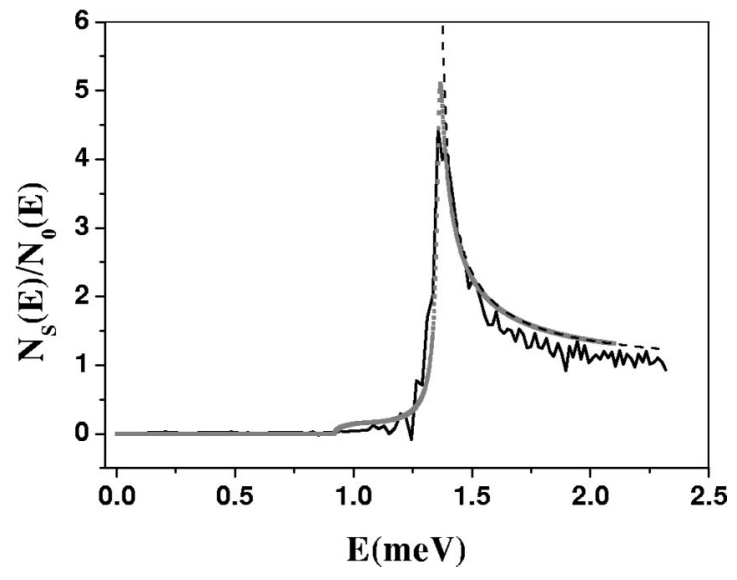

FIG. 3. Density of states (squares) and tunneling density of states (straight line) of the intermediate electrode on the $\mathrm{Nb}$ side. The BCS density of states (dashed line) has been plotted as a comparison.

equations by assuming the estimated values $\gamma_{m}$ and $\gamma_{B}$ parameters. In Figs. 4(a) and 4(b) numerical results for DOS and the pair potential are plotted. The same procedure used both for the determination of DOS and the pair potential spatial dependence was also adopted in the presence of a steady-state current injection flowing into the intermediate electrode. As an example, in Figs. 5(a) and 5(b) experimental results for DOS in the $\mathrm{Al}$ side and the pair potential along the intermediate electrode are shown. It is interesting to note that steady-state injection tends to reduce both the jump in the pair potential at the interface and the energy gap in the $\mathrm{Nb}$ side close to the interface. Moreover, the quasiparticle injection produces effects both in DOS and the pair potential, which are described in terms of higher values of $\gamma_{m}$ and $\gamma_{B}$. In particular, the $\gamma_{m}$ value increases significantly up to 2 .

\section{DISCUSSION}

The $I_{c}$ vs $I_{\text {inj }}$ curves plotted in Fig. 2 cannot be completely described in terms of a pure thermal model based on a higher effective temperature $T^{*}$ of the detector following the current injection. In fact, we estimated $T^{*}$ by comparing the detector $I-V$ characteristics in the presence of injection to those measured at higher bath temperatures. Afterward, the estimated values of $T^{*}$ together with the $\gamma_{m}$ and $\gamma_{B}$ parameters previously determined, were used in Eq. (1) in order to evaluate the corresponding theoretical behavior of the Josephson current in the presence of the proximity effect. Experiments and theoretical predictions are compared in Fig. 6. The high degree of discrepancy indicates the failure of a simple heating model, and it suggests an interpretation of the results in term of nonequilibrium processes.

We propose a simple model to explain the observed Josephson current modification. The injected current produces a nonequilibrium state which can be taken into account through a Boltzman-type modification of the quasiparticle distribution function, ${ }^{10}$ which satisfies the kinetic Eliashberg microscopic equation, i.e., $f(\varepsilon)=f_{0}(\varepsilon)$ $+c(\mathbf{r}, t) e^{-(\varepsilon-\Delta) / K_{B} T}$. It is possible to demonstrate ${ }^{11}$ that the 

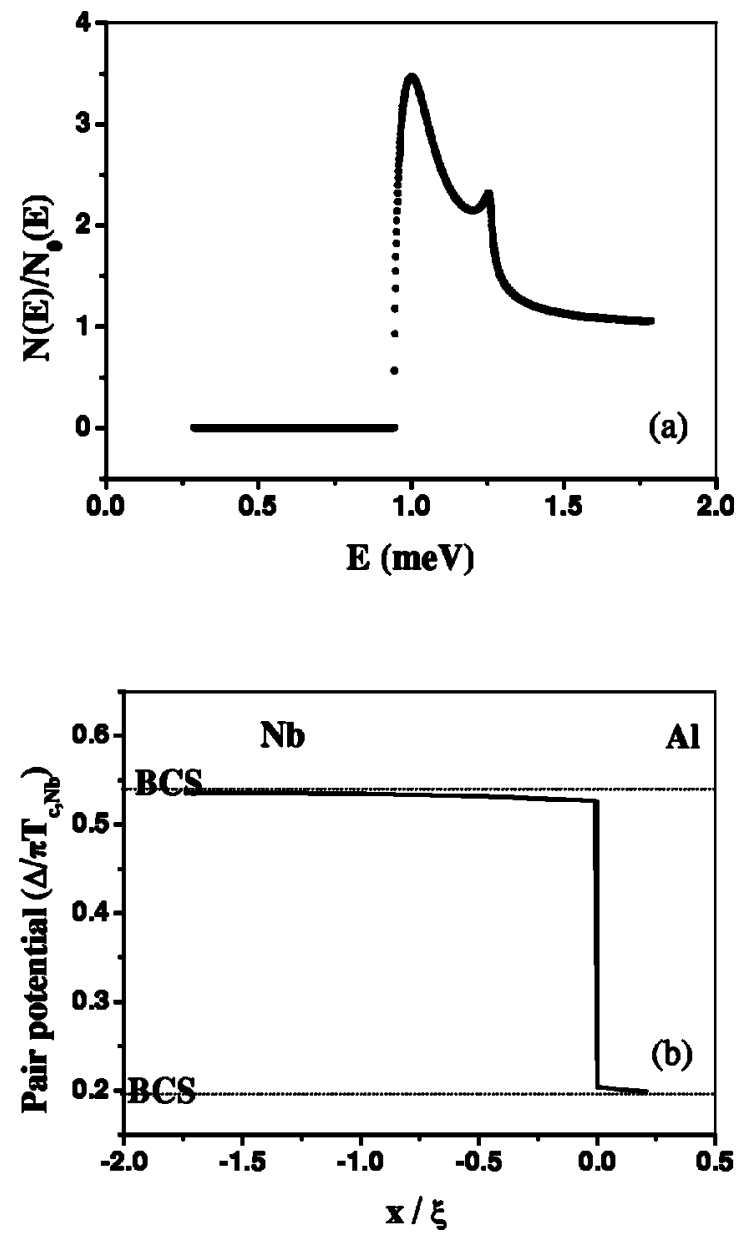

FIG. 4. (a) Numerical DOS in the Al side of the bilayer. (b) Spatial dependence of the pair potential in $\mathrm{Nb}(x<0)$ and $\mathrm{Al}(x$ $>0$ ) for the $\mathrm{Nb} / \mathrm{Al}$ bilayer at a reduced temperature $t=0.42$. $Y$ scale and $X$ scale are normalized to the critical temperature of Nb layer and to the coherence length in each layer of the bilayer, respectively.

function $c(\mathbf{r}, T)$ satisfies the following equation:

$$
\frac{\partial c}{\partial t}-D \nabla^{2} c+\frac{1}{\tau_{R}}\left[c+\frac{1}{2} \exp \left(\frac{\Delta}{k T}\right) c^{2}\right]+\frac{c}{\tau_{T}}=I_{0},
$$

where $D$ is effective diffusion constant for quasiparticles, $I_{0}$ the total injection rate, and $\tau_{T}$ and $\tau_{R}$ are the tunneling and the recombination time, ${ }^{12}$ respectively. Assuming $\tau_{T}$ $=4 e^{2} d N_{0} R_{N N}$, where $N_{0}$ is the single-spin density of states at the Fermi level, we found $\tau_{T}=3 \mu \mathrm{s}$. The determination of the thermal recombination time is much more complicated in the presence of large inhomogeneities due to proximity effect into the intermediate electrode. According to Ref. 12, we estimated the recombination time for $\mathrm{Nb}$, by assuming $\Delta_{S / N}=1.35 \mathrm{meV}$ as suggested by the above reported DOS analysis, $T=1.5 \mathrm{~K}, T_{c}=9.2 \mathrm{~K}$, and $\tau_{R}=0.3 \mu \mathrm{s}$. Nevertheless, this value must be considered only an upper limit since the proximity effect can strongly reduce $\mathrm{it}^{13}$ as explained in the following. Equation (2) is similar to the Rothwarf-Taylor equation ${ }^{14}$ for quasiparticles under the hypothesis that $2 \Delta_{1}$ phonons are at equilibrium. Since the characteristic time
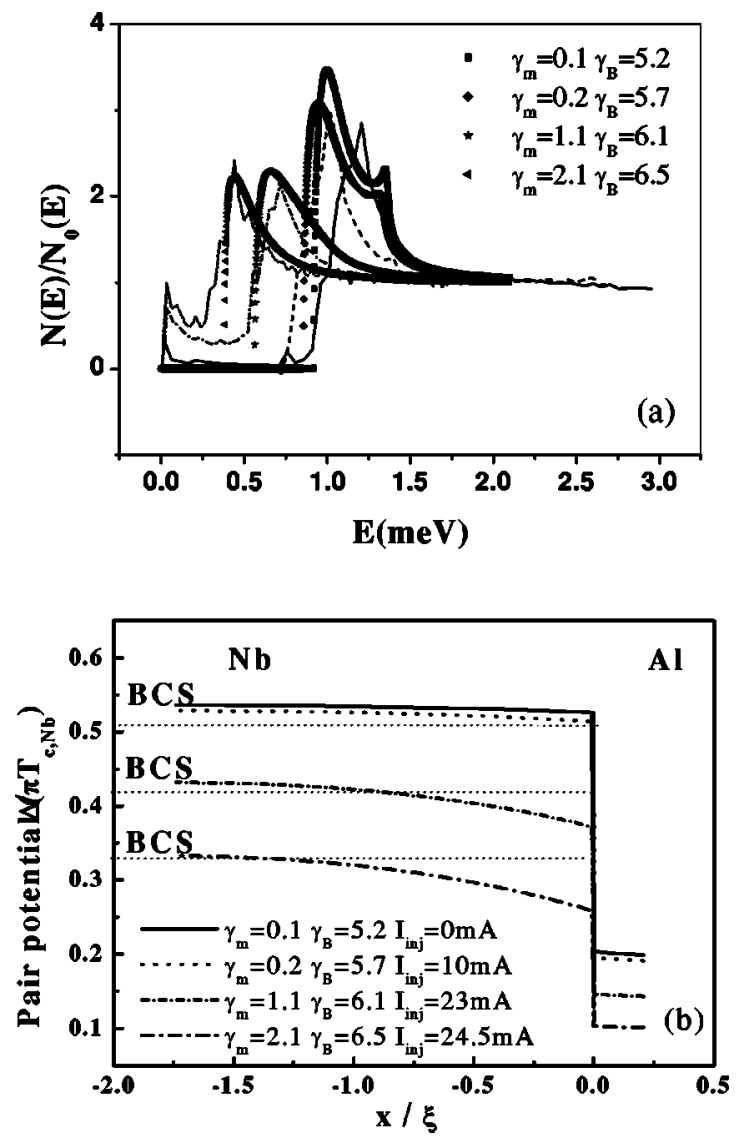

FIG. 5. (a) DOS in the $\mathrm{Al}$ side at different injection currents $I_{\mathrm{inj}}=0 \mathrm{~mA}$ (solid), $I_{\mathrm{inj}}=10 \mathrm{~mA}$ (dot), $I_{\mathrm{inj}}=23 \mathrm{~mA}$ (short dash dot), $I_{\mathrm{inj}}=24.5 \mathrm{~mA}$ (dash dot line). (b) The spatial dependence of the pair potential along the $\mathrm{Nb} / \mathrm{Al}$ bilayer at different injection currents $I_{\text {inj }}$ $=0 \mathrm{~mA}$ (solid), $I_{\mathrm{inj}}=10 \mathrm{~mA}$ (dot), $I_{\mathrm{inj}}=23 \mathrm{~mA}$ (short dash dot), $I_{\text {inj }}=24.5 \mathrm{~mA}$ (dash dot line).

scale for phonon interactions is typically 2 or 3 orders of magnitude shorter than that involving quasiparticles, the perturbed superconductor can be considered as a two component system in which only quasiparticles are out-of equilibrium with the low-temperature phonon bath.

By averaging over the volume of the perturbed electrode and by assuming steady-state conditions, Eq. (2) becomes

$$
\frac{\langle c\rangle}{\tau_{\mathrm{qp}}}=I_{0},
$$

where $\tau_{\mathrm{qp}}$ is the effective quasiparticle lifetime and $\langle c\rangle$ represents the averaged value of the $c(\mathbf{r}, t)$ function on the perturbed electrode, i.e., $c=\langle c(r)\rangle$. The diffusion term does not appear due to the boundary conditions on $c(\mathbf{r}, t)$, i.e., $\nabla c$ $\cdot n=0$. In terms of $\langle c\rangle$ the variation of the Josephson critical current can be written in the form (see Ref. 4)

$$
\frac{I_{c 0}-I_{c}}{I_{c 0}}=\langle c\rangle
$$

Equation (4) has been obtained by considering a symmetric injection junction, i.e., the gap parameter was assumed to be the same between the $\mathrm{Nb}$ base electrode and the $\mathrm{Nb}$ layer 


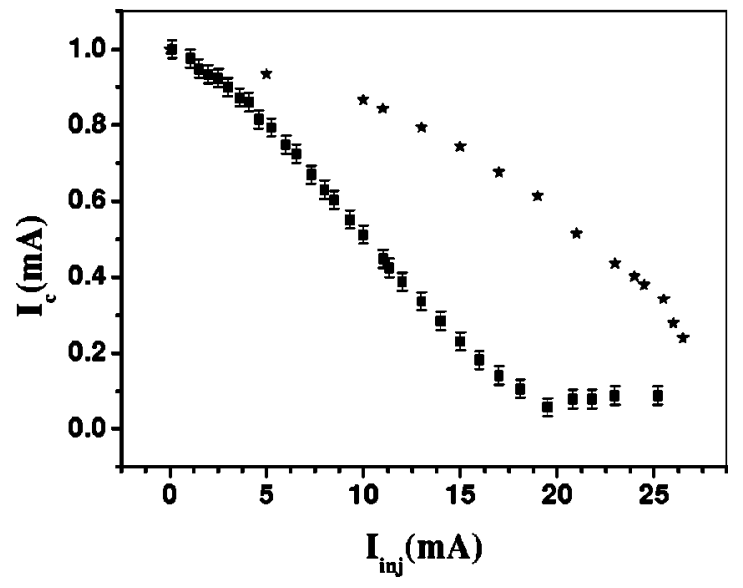

FIG. 6. The critical current $I_{c}$ as a function of the injection current $I_{\text {inj }}$ at $T=4.2 \mathrm{~K}$ (squares) is compared to the $I_{c}$ evaluated from the microscopic proximity model (stars) as a function of the effective temperature obtained from thermal dependence of detector $I-V$ characteristics.

close to the injector barrier. This assumption is really acceptable since the $I-V$ curve of the injector is almost equal to that of a standard $\mathrm{Nb}-\mathrm{Al}_{x} \mathrm{O}_{y}-\mathrm{Nb}$ superconducting tunnel junction.

The total driving term $I_{0}$ represents the number of injected quasiparticles $N$ which tunnel across the injector per unit time. A simple expression for this quasiparticle number $N$ can be derived on the basis of simple physical assumptions. In fact, the number of quasiparticles which cross over the barrier at a given $I_{\text {inj }}$ is about $e V_{\text {inj }} / \Delta_{S N}$, where $V_{\text {inj }}$ is the voltage applied to the injector. The energy transferred to the intermediate electrode by injection is almost $\left(e V_{\text {inj }}\right)^{2} / \Delta_{S N}$. By assuming the energy conservation during relaxation processes of quasiparticles down to the energy gap $\Delta_{S N}$, i.e., the limiting energy for the quasiparticles in the intermediate electrode on the $\mathrm{Nb}$ side before recombination occurs, we can write the following energy balance $N \Delta_{S N}$ $\approx\left(e V_{\text {inj }}\right)^{2} /\left(\Delta_{S N}\right)$, and hence $N=\left(e V_{\text {inj }}\right)^{2} / \Delta_{S N}^{2}$. As a consequence, the total injection rate will be given by $I_{0}$ $=\left(1 / \tau_{T}\right)\left[\left(e V_{\text {inj }}\right)^{2} / \Delta_{S N}^{2}\right]$. By inserting this expression into Eq. (3) we obtain the steady-state solution for the function $\langle c\rangle=\left(\tau_{\mathrm{qp}} / \tau_{T}\right)\left[\left(e V_{\mathrm{inj}}\right)^{2} / \Delta_{S N}^{2}\right]$, and finally the normalized Josephson current variation

$$
\frac{I_{c 0}-I_{c}}{I_{c 0}}=\frac{\tau_{\mathrm{qp}}}{\tau_{T}}\left(\frac{e V_{\mathrm{in}}}{\Delta_{S N}}\right)^{2}
$$

In Fig. 7 the experimental normalized Josephson current variation after the background subtraction as a function of $\left(e V_{\text {inj }} / \Delta_{S N}\right)^{2}$ is plotted. According to Eq. (5) the region corresponding to $e V_{\text {inj }}>4 \Delta_{S / N}$ has been fitted by a linear function, and the estimated value of the effective quasiparticle relaxation time $\tau_{\mathrm{qp}}=1.0 \pm 0.5 \mathrm{~ns}$ was obtained. This lifetime is much shorter than that predicted for $\mathrm{Nb}$ in the case of pure thermal recombination (see Ref. 12). Nevertheless, various physical effects can influence the effective recombination time of excited quasiparticles such as the proximity coupling between the $\mathrm{Nb}$ and the $\mathrm{Al}$ layers in the intermediate elec-

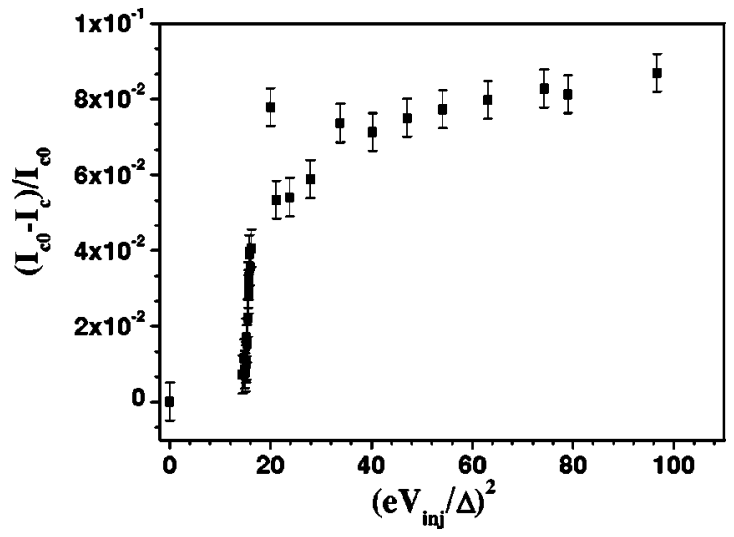

FIG. 7. The normalized Josephson current variation as a function of the excess number of injected quasiparticle.

trode, the quasiparticle self-recombination process, and the dependence of the recombination time on the perturbed film thickness.

In the presence of a proximity effect, the number of occupied states-mainly those with energies less than $\Delta_{S / N}$-increases, giving rise to a smaller $\tau_{R}$. Moreover, the presence of a steady-state injection current leads to an increase of the $\gamma_{m}$ parameter of the bilayer up to a value 2, as evidenced in Fig. 5. This effect is almost equivalent to an increase of the adjacent normal layer thickness without any appreciable variation of the interface transparency. According to normalized curves for recombination rates in the $S$ side plotted in Ref. 13, an increase of $\gamma_{m}$ up to 2 leads to a $\tau_{R} / \tau_{0}$ ratio of $\approx 30$ at a reduced temperature $t=0.17$. Since the characteristic time $\tau_{0}$ for $\mathrm{Nb}$ is $0.15 \mathrm{~ns}$, the effective quasiparticle lifetime can reduce to about $5 \mathrm{~ns}$ in our devices. On the other hand, the quasiparticle self-recombination ${ }^{15}$ seems not to be effective in our experiments. Indeed, the number density of excess quasiparticles $n_{\mathrm{exc}}=I_{\mathrm{inj}} \tau_{T} /(\mathrm{eV})$ is much smaller than the number density of thermal quasiparticles $n_{T}=4 N(0) \Delta_{0} K_{1}\left(\Delta_{0} / k_{B} T\right)$, where $K_{1}(x)$ is the secondorder modified Bessel function. In particular, $n_{\text {exc }}$ $=I_{\mathrm{inj}} \tau_{T} /(e V)=1 \times 10^{11} \mathrm{~cm}^{-3}$ and $n_{T}=1.6 \times 10^{19} \mathrm{~cm}^{-3}$, and hence the correction to the quasiparticle recombination rate due to $n_{\text {exc }}$, proportional to $\left(n_{\text {exc }} / n_{T}+2\right)$, is not effective. Moreover, the dependence on film thickness of $\tau_{R}^{-1}$, which is related to the existence of some limitations of the phonons inside the perturbed film, ${ }^{16}$ can be responsible for a further shortening up to a factor 2 . In fact, in the limit of small thickness $d \ll v_{p h} \tau_{\mathrm{PB}}$, where $\tau_{\mathrm{PB}}$ is the pair-breaking time, we found $\left(\tau_{R}^{\text {eff }}\right)^{-1} \approx\left[1-\left(2 d / v_{p h} \tau_{\mathrm{PB}}\right)\right]\left(\tau_{R}\right)^{-1}$, and hence $\left(\tau_{R}^{\text {eff }}\right)^{-1} \cong 0.5\left(\tau_{R}\right)^{-1}$ by assuming typical values for $\tau_{\mathrm{PB}}$ $\approx 10^{-10} \mathrm{~s}$ and $v_{p h} \approx 10^{3} \mathrm{~m} / \mathrm{s}$, respectively. Within these corrections, the quasiparticle lifetime estimated by measuring the Josephson current variation in steady-state injectiondetection experiments is anyway consistent with theoretical predictions expected for proximized Nb/Al bilayers. ${ }^{17}$ The simple model we proposed confirms the quasiparticles relax mainly in the reduced gap region of the $\mathrm{Nb}$ layer inside the intermediate electrode. This process strongly limits the quasiparticle lifetime, and hence the number of injected quasiparticles which reach the detector tunnel barrier and produce 
a change in the Josephson current. The consistency of this result with the expected quasiparticle trapping in $\mathrm{Nb} / \mathrm{Al}$ proximized bilayers is an indirect demonstration of the nonequilibrium nature of the observed phenomenology.

\section{CONCLUSIONS}

In conclusion, we investigated the role of a proximized $\mathrm{Nb} / \mathrm{Al}$ bilayer, which forms the intermediate electrode of a stacked junction device, in determining the nonequilibrium behavior of the Josephson current of one of the two junctions under a steady-state current injection from the other one. The spatial profile of the pair potential along the common electrode and the effective density of states (DOS) close to the detector tunnel barrier have been determined with and without the current injection, by combining the deconvolution of the current-voltage $I-V$ characteristics of the injector and the numerical solution of microscopic proximity equations. A simple nonequilibrium model for the Josephson current variation in the presence of a steady-state current injection has been also proposed. The model assumes the quasiparticle lifetime $\tau_{\mathrm{qp}}$ in the $\mathrm{Nb} / \mathrm{Al}$ intermediate electrode as a free parameter. By comparing experiments to theoretical predictions we estimated $\tau_{\mathrm{qp}}=1.0 \pm 0.5 \mathrm{~ns}$. This relaxation time, which limits the quasiparticle scattering processes in the proximized $\mathrm{Nb} / \mathrm{Al}$ bilayer, is consistent with the effective quasiparticle trapping in the $\mathrm{Nb}$ side of the intermediate electrode as also evidenced by detection experiments employing superconducting tunnel junction detectors.

\section{ACKNOWLEDGMENTS}

This work was supported by MURST in the framework of the COFIN2001 Program "Technology and applications of cryogenic microdetectors." We are grateful to M. Russo and C. Granata for device fabrication. We are indebted to Professor B. Ivlev for useful discussions and suggestions. Special thanks to Dr. F. Lombardi for a careful reading of the manuscript and useful hints.
${ }^{1}$ N. E. Booth, P. A. Fischer, M. Nahum, and J. N. Ullom, Supercond. Sci. Technol. 12, 538 (1999).

${ }^{2}$ G. Carapella and G. Costabile, Appl. Phys. Lett. 72, 377 (1998).

${ }^{3}$ Nonequilibrium Superconductivity, Phonons, and Kapitza Boundaries, edited by K. E. Gray (Plenum, New York, 1980); G. P. Pepe, G. Ammendola, G. Peluso, A. Barone, L. Parlato, E. Esposito, R. Monaco, and N. E. Booth, Appl. Phys. Lett. 77, 447 (2000).

${ }^{4}$ G. P. Pepe, G. Ammendola, G. Peluso, A. Barone, E. Esposito, L. Parlato, and B. Ivlev, Phys. Rev. B 60, 13131 (1999).

${ }^{5}$ N. E. Booth, Appl. Phys. Lett. 50, 293 (1987).

${ }^{6}$ G. P. Pepe, G. Peluso, R. Scaldaferri, L. Parlato, C. Granata, E. Esposito, and M. Russo, Eur. Phys. J. B 23, 421 (2001).

${ }^{7}$ A. Barone and G. Paternò, in Physics and Applications of the Josephson Effect (Wiley, New York, 1982).

${ }^{8}$ A. A. Golubov, E. P. Houwman, J. G. Gijsbertsen, V. M. Krasnov,
J. Flokstra, H. Rogalla, and M. Yu. Kuprianov, Phys. Rev. B 51, 1073 (1995).

${ }^{9}$ M. Blamire, Physica C 211, 467 (1993).

${ }^{10}$ R. A. Vardanyan and B. I. Ivlev, Sov. Phys. JETP 38, 1156 (1974).

${ }^{11}$ B. I. Ivlev, G. P. Pepe, and U. Scotti di Uccio, Nucl. Instrum. Methods Phys. Res. A 300, 127 (1991).

${ }^{12}$ S. B. Kaplan, C. C. Chi, D. N. Langenberg, J. J. Chang, S. Jafarey, and D. J. Scalapino, Phys. Rev. B 14, 4854 (1976).

${ }^{13}$ A. A. Golubov, E. P. Houwman, J. G. Gijsbertsen, J. Flokstra, H. Rogalla, J. B. le Grand, and P. A. J. de Korte, Phys. Rev. B 49, 12953 (1994).

${ }^{14}$ A. Rothwarf and B. N. Taylor, Phys. Rev. Lett. 19, 27 (1967).

${ }^{15}$ P. Verhoeve, N. Rando, J. Verveer, A. Peacock, A. van Dordrecht, P. Videler, and M. Bavdaz, Phys. Rev. B 53, 809 (1996).

${ }^{16}$ W. Eisenmenger and A. Daym, Phys. Rev. Lett. 18, 125 (1967).

${ }^{17}$ A. A. Golubov and E. P. Houwman, Physica C 205, 147 (1993). 